\title{
Proposta interdisciplinar de apoio à cessação do tabagismo em uma unidade de saúde da Estratégia Saúde da Família: relato de experiência
}

\author{
Interdisciplinary proposal for tobacco cessation at a primary health care unit: an experience report
Propuesta interdisciplinaria para cese del uso de tabaco en una unidad de salud de la Estrategia de Salud Familiar: relato de experiencia

Rafael Silva Duarte. Universidade Federal do Rio de Janeiro (UFRJ). Rio de Janeiro, RJ, Brasil. rafaelsilvaduarte@gmail.com (Autor correspondente) Isa Haro Martins. Escola Nacional de Saúde Pública (ENSP). Rio de Janeiro, RJ, Brasil. isa.haro.m@gmail.com

Cristiane Pereira Mendes. Escola Nacional de Saúde Pública (ENSP). Rio de Janeiro, RJ, Brasil. cris.p.mendes@uol.com.br Maria Antônia Silva Costa. Escola Nacional de Saúde Pública (ENSP). Rio de Janeiro, RJ, Brasil. mariantoniasilvacosta@gmail.com Daniela de Souza Mendes. Escola Nacional de Saúde Pública (ENSP). Rio de Janeiro, RJ, Brasil. dani.danifisio@hotmail.com Valéria Ferreira Romano. Universidade Federal do Rio de Janeiro (UFRJ). Rio de Janeiro, RJ, Brasil. valromano@uol.com.br Sérgio Coelho Gomes. Universidade Federal do Rio de Janeiro (UFRJ). Rio de Janeiro, RJ, Brasil. scoelhogomes@gmail.com Alessandra Reis. Escola Nacional de Saúde Pública (ENSP). Rio de Janeiro, RJ, Brasil. alessandra291274@yahoo.com.br Regina Dias Neves. Escola Nacional de Saúde Pública (ENSP). Rio de Janeiro, RJ, Brasil. reginadneves@gmail.com

\section{Resumo}

Este artigo descreve a proposta interdisciplinar utilizada atualmente no Centro Municipal de Saúde Manguinhos (CMSM), Rio de Janeiro, para condução longitudinal do tratamento antitabagismo em pacientes residentes em comunidades de baixa renda da região, sob a ótica de um estudante de medicina em seu período de internato rotatório. A abordagem antitabágica consiste em terapia longitudinal dividida em duas etapas: (i) assistência a grupo de pacientes com abordagem interdisciplinar ao longo de quatro sessões distribuídas semanalmente que inclui tratamento psicoterápico e recursos farmacológicos; seguida de (ii) duas sessões quinzenais de terapia de manutenção, caracterizadas por atenção individualizada e desmame farmacológico, complementadas por um seguimento mensal de até um ano. Os protocolos atuais, as atividades dos profissionais e a condução da proposta são descritos. Este relato sugere que o aprimoramento da formação em medicina pode ocorrer por meio da participação dos estudantes em ações de educação em saúde, tais como o grupo antitabagismo.

\begin{abstract}
This article describes the interdisciplinary approach currently used at the Manguinhos Municipal Health Centre (MMHC), Rio de Janeiro, for the longitudinal management of anti-smoking treatment in patients living in low-income community regions, from the perspective of a medical student in his rotating internship. The anti-tobacco approach consists in a longitudinal therapy divided in two steps: (i) four interdisciplinary anti-tobacco group sessions scheduled weekly, which includes psychotherapy and pharmacological resources; followed by (ii) two sessions of maintenance therapy scheduled fortnightly, characterized by individualized care and pharmacological withdrawal, complemented by a monthly one year follow-up. This article describes the current protocols, the professional activities and how the proposal was developed. This report suggests that improvement in medical training might occur through participation of medical students in health education activities such as tobacco cessation group.
\end{abstract}

\section{Resumen}

En este artículo se describe el enfoque interdisciplinario utilizado actualmente en el Centro Municipal de Salud Manguinhos (CMSM), Río de Janeiro, para la conducción longitudinal del tratamiento anti-tabaquismo en los pacientes que residen en comunidades de bajos ingresos de la región, desde la perspectiva de un estudiante de medicina en su período de internado rotatorio. La abordaje anti-tabaquismo consiste en una terapia longitudinal dividida en dos etapas: (i) asistencia al grupo de pacientes con un enfoque interdisciplinario distribuidas en cuatro sesiones semanales que incluyen psicoterapia y recursos farmacológicos; seguido por (ii) dos sesiones quincenales de terapia de mantenimiento caracterizadas por la atención individualizada y retirada del medicamento, complementadas con un seguimiento mensual hasta un año. Son presentados protocolos actualizados y las actividades de los profesionales y como las actividades son desarrolladas. Este relato sugiere que la mejora de la formación médica puede ocurrir a través de la participación estudiantil en las acciones de educación en salud, tales como el grupo antitabaco.

Como citar: Duarte RS, Martins IH, Mendes CP, Costa MAS, Mendes DS, Romano VF et al. Proposta interdisciplinar de apoio à cessação do tabagismo em uma unidade de saúde da estratégia saúde da família: relato de experiência. Rev Bras Med Fam Comunidade. 2014;9(33):pp-pp. Disponível em: http://dx.doi.org/10.5712/rbmfc9(33)708
Palavras-chave:

Hábito de Fumar Abandono do Uso de Tabaco Atenção Primária à Saúde Educação Médica

\section{Keywords:}

Smoking

Tobacco Use Cessation Primary Health Care Education Medical

\section{Palabras clave:} Hábito de fumar Cese del Uso de Tabaco Atención Primaria de Salud Educación Médica

\section{Fonte de financiamento:} declaram não haver. Parecer CEP: não se aplica.

Conflito de interesses: declaram não haver. Recebido em: 27/02/2013. Aprovado em: 02/03/2014. 


\section{Introdução}

A nicotina consiste em um dos agentes psicoativos de maior consumo em diferentes países. ${ }^{1,2,3} \mathrm{O}$ ato de fumar cigarros, também denominado tabagismo, é uma das grandes causas de morte por doença com possibilidade de serem prevenidas em todo o mundo. ${ }^{1,2,4,5}$ Estima-se que um em cada 10 óbitos entre adultos seja atribuível às doenças relacionadas ao tabaco, totalizando aproximadamente 5,4 milhóes de óbitos anuais, ${ }^{2,5,6}$ sendo que cerca de $80 \%$ dessas mortes ocorrem em países em desenvolvimento. O tabagismo está composto por dois pilares: (i) dependência da nicotina e (ii) doenças relacionadas ao consumo do tabaco. ${ }^{3,4} \mathrm{O}$ tabagismo favorece especialmente a emergência de doenças cardiovasculares, respiratórias e na cavidade oral, além de patologias em outros órgãos e sistemas. ${ }^{1,2,3,5}$

As medidas de intervenção estão amplamente disponíveis e acessíveis, porém são subutilizadas devido ao uso liberado da nicotina e à aceitação cultural do tabagismo. ${ }^{7}$ Médicos, especialmente na anamnese para indivíduos aparentemente sãos, costumeiramente não reforçam a importância do tabaco no futuro fisiopatológico do fumante ou desconhecem o tratamento para sua cessação. Acredita-se que a pressão para redução no tempo de consulta e a descrença nos benefícios reais da cessação do tabagismo contribuem para a não aplicação de medidas intervencionistas. ${ }^{5,6,7} \mathrm{O}$ atendimento ambulatorial geralmente prioriza resultados em curto prazo e, no caso do tabagismo, as pessoas que possuem esse hábito só iráo sucumbir aos seus efeitos muitos anos depois, sendo esses efeitos frequentemente invisíveis. Todos esses fatores fazem com que haja pouco direcionamento do atendimento ambulatorial para a abordagem do tabagismo. , $3,5,7,8,9^{-1}$

Escolas e universidades, em território brasileiro e em diferentes países, não dispóem de programas regulares efetivos para a abordagem do tabagismo, e os profissionais de saúde nelas formados recebem pouco treinamento sobre esse tema, contabilizando, em geral, menos de cinco horas de formaçáa para tratamento da dependência do tabaco. . $2,3,5,8,10,11,12^{2}$

Médicos que atuam na Atenção Primária à Saúde (APS) desempenham papel fundamental no diagnóstico, na abordagem e no tratamento de fumantes. Estima-se que aproximadamente $70 \%$ dos tabagistas recebam atendimento em saúde, o que significa uma excelente oportunidade para que os profissionais médicos da APS possam, em cada visita, intervir a favor da cessaçáo do tabagismo. Recomendaçôes breves ou encorajamento durante uma visita médica resultam em uma taxa de 5\% de abandono do tabagismo, sem reincidências em pelo menos um ano. Taxas superiores são atingidas quando programas ou estratégias de maior qualidade são elaborados e implantados. Programas personalizados resultam em tabagistas que evoluem para a cessação do tabagismo por mais de cinco anos. ${ }^{1,3,9,13}$ Por outro lado, não há dados quanto aos impactos da abordagem interdisciplinar e sobre a constituição das equipes envolvidas.

Atualmente, uma parceria entre a Faculdade de Medicina da Universidade Federal do Rio de Janeiro (UFRJ) e diferentes unidades da Estratégia Saúde da Família (ESF) do estado do Rio de Janeiro, incluindo o Centro Municipal de Saúde Manguinhos (CMSM), tem possibilitado o estágio prático dos alunos de medicina durante o internato rotatório (08 semanas) e eletivo (06 meses) em Medicina de Família e Comunidade (MFC). Além de outros objetivos e possibilidades, tais períodos permitem o aprofundamento do conhecimento do aluno em relação à promoção da saúde, especialmente em estratégias antitabagismo. Durante o período do estágio, o interno em medicina na unidade CMSM tem a possibilidade de desenvolver semanalmente atividades teóricas e práticas com pacientes e equipe do grupo antitabagismo, resultando, dessa forma, no enraizamento de conceitos e condutas que permitam a aplicação desses métodos após a conclusão do curso.

Considerando esses aspectos, e fundamentando-se nas diretrizes descritas em diferentes países, o objetivo deste artigo é, sob a visão do estudante acadêmico de medicina - em seu internato rotatório durante 02 meses de atividades semanais no grupo antitabagismo - apresentar a proposta interdisciplinar antitabagismo implementada no CMSM Manguinhos, Rio de Janeiro, no contexto da ESF. A organização em questão foi denominada "proposta interdisciplinar CMSM" de forma a registrar uma identidade ao processo apresentado. A proposta consiste em uma adaptação da estratégia divulgada pelo Ministério da Saúde (MS), a qual carece de orientaçóes, detalhamentos e estudos de implementação específicos para a ESF.

\section{Descrição da experiência}

A descrição, análise e revisão da literatura foram realizadas no período de julho a setembro de 2012 pelo autor principal do presente artigo, estudante de medicina da Universidade Federal do Rio de Janeiro (UFRJ), em seu período de internato rotatório (oito semanas) em MFC no CMSM. Durante tal período, o aluno frequentou as atividades semanais do grupo antitabagismo, participando ativamente das entrevistas, terapias de grupo, reunióes e condutas sob supervisão dos médicos 
e enfermeiros responsáveis. Tais experiências foram devidamente registradas por meio de anotaçóes e relatos descritivos, discutidas com os profissionais do serviço, e analisadas comparativamente com a literatura vigente, resultando neste artigo com informaçôes atualizadas para acadêmicos de medicina e profissionais de saúde em formação. No período do estágio, foram assistidos 30 pacientes em dois ciclos de seis sessôes de tratamento, com dados preliminares de 75\% de adesão ao tratamento nesse curto período. O presente relato não recebeu financiamento de quaisquer fontes ou instituiçóes envolvidas no trabalho, e não há conflito de interesses.

\section{A proposta interdisciplinar CMSM e o papel dos profissionais}

A proposta interdisciplinar CMSM foi iniciada em junho de 2012, no Centro Saúde-Escola Germano Sinval Faria (CSEGSF), uma das unidades do CMSM localizada na Fundação Oswaldo Cruz (FIOCRUZ), responsável pela assistência à população de Manguinhos, localizada na zona norte da cidade do Rio de Janeiro-RJ, Brasil, por meio da iniciativa Território Integrado de Ação à Saúde - TEIAS-Escola Manguinhos. A proposta vigente é constituída por profissionais interessados em atuar na causa antitabágica, reunidos de maneira voluntária, com foco na terapêutica antitabágica de grupos de pacientes, podendo pertencer tanto a diferentes equipes de ESF como ao Núcleo de Apoio à Saúde da Família (NASF). Na unidade CMSM, médicos, enfermeiros, farmacêuticos, fisioterapeuta, educador físico, agentes comunitários de saúde (ACS), nutricionista e odontólogo são os profissionais atuantes (Figura 1).

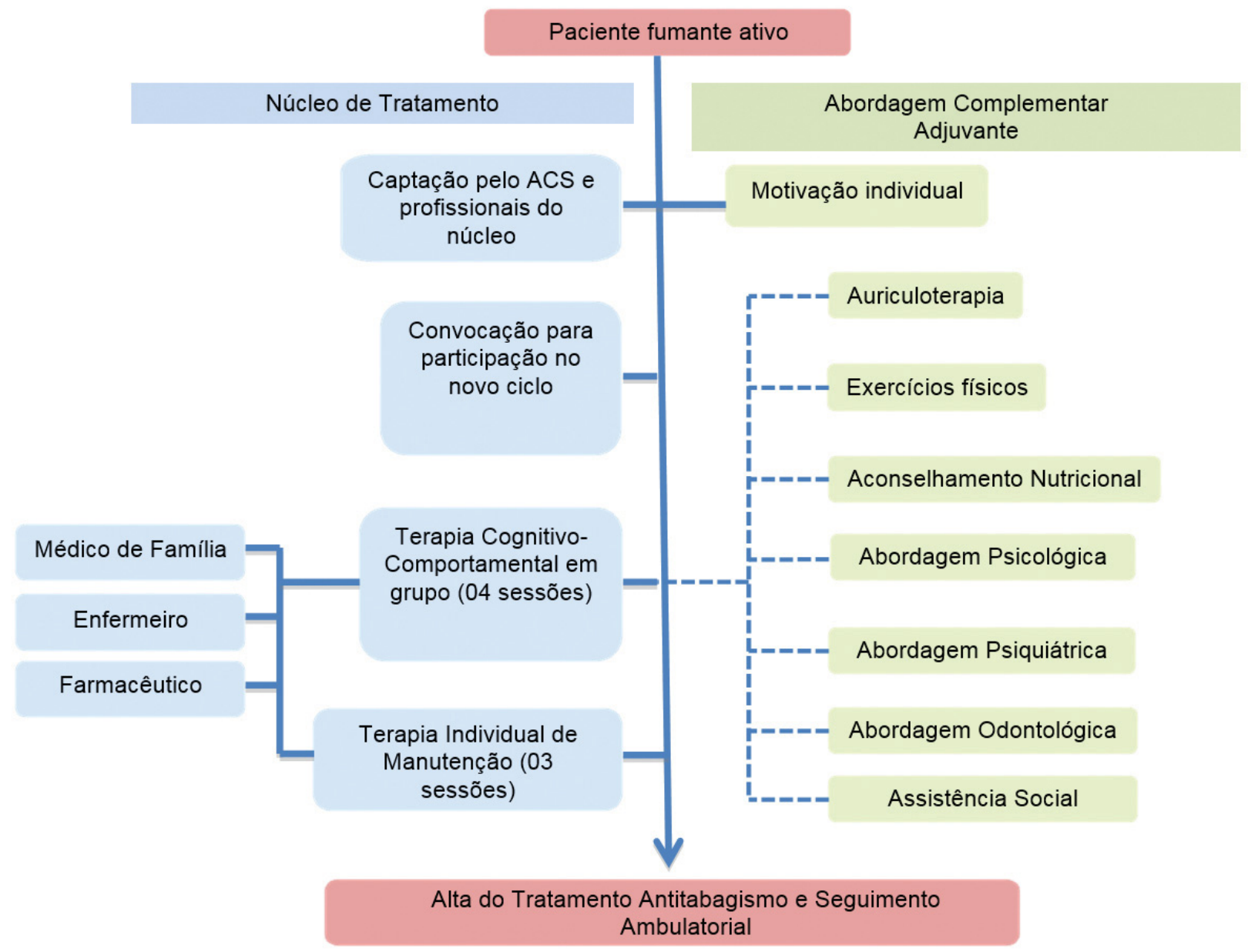

O tracejado indica possibilidades de açóes especiais, mediante a disponibilidade institucional no período requerido pela equipe do CMSM.

Figura 1. Estrutura organizacional da proposta interdisciplinar CMSM para tratamento antitabagismo. 
A proposta vigente apresenta um cronograma fixo inicial composto por seis sessôes, e um seguimento mensal até um ano. As sessôes iniciais têm duração aproximada de duas horas e se distribuem em duas fases: (a) Terapia Cognitivo-Comportamental (TCC) em grupo com quatro sessôes semanais; e (b) Terapia Individual de Manutenção (TIM) com duas sessôes quinzenais. Em todas as sessóes os pacientes têm seus sinais vitais aferidos pelos profissionais envolvidos capacitados, são submetidos à auriculoterapia, e o tratamento farmacológico é oportuno e individualizado.

O grupo antitabagismo é aberto a toda a população da área de Manguinhos. A pré-inscrição do paciente é feita de maneira espontânea, por contato direto do mesmo com os membros da equipe da ESF, e registro de telefone. Os interessados são contatados posteriormente para divulgação do período de inscrição e da programação. A demanda espontânea na inscrição também é aceita. Os pacientes inscritos na lista de espera têm prioridade no novo ciclo de tratamento. Sáo incluídos fumantes ativos, com ou sem diagnóstico de doenças relacionadas ao tabaco, em recaída ou sem tentativa anterior, sem discriminação de carga tabágica, sexo, cor, idade ou comorbidades. As sessóes são realizadas no período diurno, em uma sala com cadeiras e mesas suficientes para boa acomodação, ar condicionado, em ambiente reservado e tranquilo. Não são admitidas faltas às sessóes semanais e quinzenais para o devido tratamento continuado. Aos pacientes que náo conseguem parar de fumar até o final da TCC em grupo é aconselhado o reinício do processo em outra oportunidade. Em cada ciclo de tratamento, novo grupo de 10 a 15 pacientes é admitido, garantindo uma frequência anual de seis a oito ciclos, com até 120 pacientes assistidos.

Terapia Cognitivo-Comportamental(TCC) em grupo: composta por todos os pacientes inscritos e realizada de forma continuada, a TCC em grupo é liderada pelos profissionais médicos e enfermeiros. É feita uma abordagem psicossocial com perguntas da equipe sobre o histórico tabagista individual, além de relatos sobre o processo de tratamento, acompanhamento médico individual para avaliação de conflitos, sintomas de abstinência, e efeitos físicos e psíquicos decorrentes do tratamento. O primeiro encontro corresponde a uma entrevista individual onde são coletados dados pessoais (sexo, idade, renda familiar, nível de escolaridade e outros), além de avaliaçáo do apoio familiar, facilidade de acesso ao centro de tratamento, anamnese clínica completa com aplicação do Questionário de Tolerância de Fagerström, avaliação do estágio da motivação, de depressão, aplicação do teste de CAGE para etilismo, avaliação de contraindicaçóes e apresentação da proposta por meio de um modelo informativo clássico. A sessão seguinte de TCC em grupo é caracterizada pela disposição dos pacientes em círculo e por perguntas e relatos individuais, assumindo uma abordagem de intervenção motivacional. Para tal, o fumante é estimulado a passar à ação, incluindo a definição da data de parar de fumar (dia "D" ou Quit Day), permitindo-se a decisão individual por diferentes formas de parada (redução gradual ou parada abrupta). O paciente é orientado sobre medidas comportamentais que o afastem da lembrança ou rotina diretamente ligada ao cigarro. Também é orientado para o combate à fissura e estratégias para manter as mãos ocupadas e evitar recaídas. Os pacientes são estimulados a estabelecerem metas alcançáveis e contextualizadas, dentro das limitaçóes de cada indivíduo. A partir da suspensão do fumo, dá-se início ao tratamento por meio de adesivos transdérmicos, na ausência de contraindicaçôes, sendo utilizadas gomas e/ou pastilhas de nicotina em casos especiais. Prefere-se a monoterapia com adesivos, devido à comprovada melhor adesão. A dose utilizada para os adesivos transdérmicos é individualizada de acordo com a carga tabágica do paciente. Cada paciente recebe a medicação correspondente a uma semana, vinculando a data de finalização da medicação à data da sessão semanal seguinte. A bupropiona somente é prescrita em casos especiais. Nas três sessóes seguintes de TCC em grupo é realizada a continuidade ao aconselhamento intensivo e intervenção motivacional, com participação de ex-fumantes partilhando suas experiências, assim como o acompanhamento do processo evolutivo do abandono da dependência do tabagismo e das reaçóes adversas. É estimulada a troca de experiências, dificuldades, tentativas e sucessos entre os fumantes.

Terapia Individual de Manutençáo (TIM): caracteriza-se por dois encontros quinzenais individuais de acompanhamento e desmame da medicaçáo. Cada encontro tem duração aproximada de 30 minutos, com uma abordagem direcionada à intervençấo motivacional. É feita a redução progressiva da dose do fármaco em uso, até o encerramento da terapia.

Seguimento: o acompanhamento posterior é realizado mensalmente, até completar um ano, em um grande grupo do qual participam todos os pacientes em processo de manutençáo. A presença nesse grupo não é obrigatória. Nesta etapa é fundamental que a equipe de ESF responsável pelo atendimento àquele paciente seja notificada de que ele se encontra em fase de manutenção, para adequado acompanhamento. Em caso de recaída, há possibilidade de encaminhamento a um novo grupo.

Nessa proposta organizacional, a interação entre os profissionais de ação fundamental (ACS, enfermeiros, médicos, farmacêuticos) e os profissionais das especialidades complementares (fisioterapeuta, educador físico, nutricionista, psiquiatra e odontólogo) favorece a continuidade e o alcance do processo na ESF. O papel de cada profissional é descrito abaixo. 


\section{Profissionais pertencentes ao núcleo antitabagismo na proposta CMSM}

Agente Comunitário de Saúde (ACS): a partir da adscrição das famílias em sua microárea, os ACS orientam os indivíduos quanto aos serviços de saúde disponibilizados (divulgação) e recrutam pacientes fumantes ativos que apresentem interesse e/ou necessidade de cessação do tabagismo. Em caso de abandono da continuidade do tratamento, os ACS se responsabilizam por avaliar e caracterizar os motivos de encerramento de vínculo, e estimulam o paciente ao retorno para reinício do tratamento em nova oportunidade. Tais funçóes também são exercidas por outros profissionais do núcleo.

Médico de Família e Comunidade (MFC): na proposta interdisciplinar CMSM, atualmente, um profissional médico atua como líder do grupo antitabagismo, junto com o profissional de enfermagem, sendo ambos responsáveis por um ciclo de tratamento. Médico e enfermeiro atuam diretamente na condução dos grupos e no trabalho psicossocial intensivo durante as sessôes. São também responsáveis pela revisão de fichas de anamnese; avaliação de comorbidades; contraindicaçôes; captação de sinais que indiquem conflitos; distúrbios psíquicos ou sintomas decorrentes da abstinência; e aplicação de medidas de intervenção para controle multissistêmico em cada caso. O médico é o responsável pelas prescrições farmacológicas e também é o condutor das terapias individualizadas, quando necessárias, ou durante o período de manutenção. É também a ele atribuída a função de detectar casos que necessitem de complementação profissional ou até mesmo atendimento em atençẫo secundária ou terciária e/ou interrupção do tratamento.

Enfermeiro: atua de forma protagonista e efetiva em parceria com o profissional médico, compartilhando de todas as atribuiçóes e açóes citadas no item anterior e assumindo a coordenação do projeto. Realiza o levantamento de dados demográficos, avaliação estatística trimestral para acompanhamento, assim como organização dos livretos com tarefas semanais.

Farmacêutico: não participa diretamente das sessóes do grupo antitabagismo, porém é parte fundamental da estrutura da ESF, que possui uma farmácia a serviço das comunidades assistidas. É o responsável pela dispensação de medicamentos, incluindo revisão das prescrições e orientaçôes aos pacientes.

\section{Profissionais complementares na proposta CMSM}

Fisioterapeuta: é responsável pela aplicação de auriculoterapia individualizada durante todas as sessões. Embora a efetividade da auriculoterapia, como coadjuvante na terapia antitabagismo para o decréscimo da ansiedade, seja estimada em aproximadamente $45 \%$, essa atividade é incluída na proposta vigente. ${ }^{14,15}$ Adicionalmente aos efeitos ansiolíticos, os pacientes demonstram satisfação e acolhimento quando submetidos à auriculoterapia, indicando possível efeito psicoterápico associado.

Educador Físico: a proposta interdisciplinar CMSM oferece aos pacientes programas de caminhadas e exercícios matinais com frequência de três manhãs por semana no território da FIOCRUZ, Rio de Janeiro-RJ. O exercício auxilia na redução do sedentarismo, auxilia aqueles pacientes com tendência a ganhar peso com a cessação do tabagismo, auxilia a diminuir a ansiedade e facilita maior convivência com o grupo de apoio. ${ }^{16} \mathrm{Tal}$ atividade é de responsabilidade do profissional de Educação Física integrante do grupo antitabagismo.

Nutricionista: importante na assistência ao paciente durante e após o tratamento antitabagismo, por meio de aconselhamento para uma reorientação alimentar, condução de programas nutricionais e dietas direcionadas e individualizadas. ${ }^{17}$

Psicólogo e Psiquiatra: devido à prevalência significativamente maior de tabagismo entre indivíduos com transtornos psiquiátricos, como depressão e abuso de álcool, é esperada a presença desses pacientes nos grupos de tratamento. É de decisão da equipe, considerando a gravidade dos distúrbios apresentados pelo paciente, que o mesmo seja inserido na proposta interdisciplinar CMSM ou receba terapia individualizada, na forma de psicoterapia e/ou acompanhamento psiquiátrico desde o início, de forma a náo comprometer o grupo e/ou o acompanhamento do paciente. É fato, contudo, que o papel do psicólogo e do psiquiatra, atuando juntos ou separadamente, na abordagem e acompanhamento dessa categoria de pacientes é essencial. ${ }^{10,18}$

Odontólogo: o encaminhamento dos pacientes às consultas por odontólogos é adequado como forma de ratificar os benefícios obtidos com a cessação do tabagismo e prevenir doenças futuras, ${ }^{3}$ além de melhorar a autoestima dos pacientes.

Assistente Social: em casos em que a abordagem familiar seja necessária, pode-se recorrer à atividade de um assistente social de forma a garantir a adesão ao tratamento. Em inúmeros casos a violência familiar, a imposição ou problemática das relaçôes afetivas domiciliares, a influência do cenário da comunidade, ou as relações ou necessidades trabalhistas, prejudicam significativamente o sucesso do tratamento antitabagismo. 


\section{Discussão}

Nos últimos anos, vários protocolos clínicos têm sido desenvolvidos em inúmeros países de acordo com as recomendaçóes consensuais para a redução do consumo do tabaco. ${ }^{1,2,3,5,8,10}$ Destacam-se as diretrizes do Reino Unido derivadas dos guidelines americanos e da organização internacional Cochrane (The Cochrane Collaboration ${ }^{\circ}$ ). No entanto, existem poucos estudos realizados na APS que direcionem para propostas efetivas de terapia em grupo na ação antitabagismo. ${ }^{2,8} \mathrm{~A}$ implementação de metodologias antitabagismo, assim como outros programas, necessita de especificaçóes precisas quanto ao tipo de população a ser incluída em determinado protocolo de tratamento, assim como possíveis necessidades de adaptaçôes e estruturação de acordo com as características da unidade de saúde, incluindo infraestrutura, disponibilidade de profissionais de saúde, ambiente e outros inúmeros fatores, os quais podem mascarar o potencial efetivo de uma proposta antitabágica.

As propostas atuais de tratamento da dependência do tabaco, incluindo as Diretrizes Brasileiras atualizadas, discorrem sobre as metodologias disponíveis e já evidenciadas, incluindo a abordagem inicial, o diagnóstico, e as variaçóes terapêuticas para o tratamento. ${ }^{10,14,19}$

Mediante a carência de propostas que incorporem as metodologias antitabagismo com evidências de eficácia comprovada e que permitam aplicaçáo na APS, no cenário da ESF, especialmente em comunidades de baixa renda - como ocorre no estado do Rio de Janeiro - este artigo descreve a proposta interdisciplinar CMSM de abordagem diagnóstica e terapêutica implementada no sistema atual local. Tal proposta não consiste de avaliação randomizada ou estudo comparativo e/ou de custo-efetividade, mas de uma iniciativa que requer adaptação necessária à implementação de protocolos gerais instituídos pelo ministério da saúde para o ambiente da APS. A forma como tal adaptação foi estruturada e é executada fornece aos pacientes uma assistência interdisciplinar ampla e agradável, podendo significar condiçóes favoráveis à adesão e efetividade da proposta. Entretanto, ainda é necessário avaliar essa proposta por meio de estudos específicos.

Os aspectos positivos observados na presente proposta incluem a possibilidade do corpo do grupo interdisciplinar antitabagismo CMSM ser composto pela soma de profissionais de diferentes equipes e do NASF, interessados na condução da atividade. A estrutura da proposta CMSM, que agrega diferentes profissionais da ESF, também possibilita açóes simultâneas em diferentes áreas da comunidade de Manguinhos. Dessa forma, transcende os limites de composiçáo de uma equipe de ESF, mas não desconfigura ou restringe o papel da mesma e sua atuação no tratamento antitabagismo individualizado. Curiosamente, tal atividade interdisciplinar agrega e proporciona maior interatividade entre as diferentes equipes de ESF que compóem a unidade de saúde, recrutando os diversos profissionais interessados, o que gradualmente possibilita a transposição dos limites geográficos, muitas vezes existentes em algumas unidades de APS.

Por outro lado, a natureza voluntária da participação dos profissionais de saúde do CMSM na proposta interdisciplinar antitabagismo torna essa proposta vulnerável, podendo comprometer a sua continuidade e o seu aprimoramento. Da mesma forma, a organização interdisciplinar atual resulta na ausência de profissionais importantes, como técnicos de enfermagem e outros, que garantiriam contribuição significativa na condução do tratamento. Adicionalmente, é importante ressaltar que a inferência dessa estrutura para outras unidades da ESF é significativamente limitada pela disponibilidade/carência de profissionais específicos, rotatividade dos possíveis profissionais interessados, limitaçôes institucionais, propriedades de cada comunidade e regiáo, entre outros aspectos. Tais consideraçôes reforçam a necessidade de desenvolvimento de estudos gradativos para o diagnóstico inicial de necessidades e possibilidades locais para implantação de programas, seguidos de estratégias diretamente relacionadas a contextos específicos em cada comunidade coberta pela ESF, de forma a permitir a elaboraçáo de propostas sítio-específicas, que respeitem a diversidade e a realidade de cada região. A presente proposta não consiste em uma estrutura ideal para implementaçáo universal, sendo uma iniciativa original e alternativa importante no programa antitabagismo no CMSM, para assistência à comunidade de Manguinhos, Rio de Janeiro.

Adicionalmente, a vivência proporcionada pela proposta interdisciplinar CMSM ao interno de medicina, em seu período de prática em MFC, permitiu a aquisição e o aprimoramento de conceitos, condutas terapêuticas e medidas de promoção da saúde, importantes para a prática médica, significativamente negligenciada em sua formação acadêmica anterior nas universidades. O período de dois meses de internato rotatório, com frequência semanal de atividades no grupo antitabagismo, foi adequado para complementação e potencialização da formação curricular básica dos novos profissionais de saúde. A estimativa é de que os profissionais envolvidos diretamente na proposta CMSM deem continuidade à prática nos próximos semestres, com abertura a novos estudantes de medicina em período de internato. Acredita-se no potencial dessa proposta como reforço ao ensino e à promoção da saúde, fornecendo simultaneamente formação médica adequada e assistência com qualidade aos pacientes. 


\section{Conclusão}

São escassos os trabalhos que destacam açóes e contribuiçóes dos profissionais de saúde no processo antitabagismo, especialmente no que tange à implementação dessas estratégias na APS. Não foram identificados na literatura científica registros sobre outras propostas de estruturação de terapia antitabágica em grupos de pacientes em serviços da APS/ESF inseridos no contexto das comunidades com baixa renda, em território brasileiro.

O estágio permitiu ao aluno de medicina, em seu período de formação prática em Medicina de Família e Comunidade (MFC), o estímulo às reflexôes, confronto e consideraçôes sobre a postura profissional e de equipes interdisciplinares na estratégia anti-tabágica. A revisão da literatura e a abordagem prática permitiram discussóes críticas ao desafio de transpor um protocolo geral para categorias específicas de populaçôes, como aquelas assistidas no CMSM Manguinhos. É significativamente importante trazer o aluno de medicina para vivenciar as dificuldades na implementação de metodologias na APS, acreditando-se na necessidade de formação de profissionais bem formados e cientes quanto à influência das diferentes realidades socioeconômicas e geográficas para a efetividade de qualquer proposta terapêutica.

\section{Referências}

1. Anczak JD, Nogler RA. Tobacco cessation in primary care: maximizing intervention strategies. Clin Med Res. 2003;1(3):201-16. http://dx.doi.org/10.3121/cmr.1.3.201.

2. Hsueh K-C, Chen C-Y, Yang Y-H, Huang C-L. Smoking cessation program in outpatients clinics of family medicine department in Taiwan: a longitudinal evaluation. Eval Health Prof. 2010;33:(1):12-25. http://dx.doi.org/10.1177/0163278709356185.

3. JCS Joint Working Group. Guidelines for smoking cessation (JCS 2010). Circ J. 2012;76(4):1024-43. http://dx.doi.org/10.1253/circj.CJ-88-0021.

4. US Public Health Service Report. A clinical practice guideline for treating tobacco use and dependence: 2008 update. Am J Prev Med. 2008;35:(2):158-76. http://dx.doi.org/10.1016/j.amepre.2008.04.009.

5. Cabezas C, Martin C, Granollers S, Morera C, Ballve JL, Zarza E, et al. Effectiveness of a stepped primary care smoking cessation intervention (ISTAPS study): design of a cluster randomized trial. BMC Public Health. 2009;9:48-59. http://dx.doi.org/10.1186/1471-2458-9-48.

6. Carson KV, Verbiest MEA, Crone MR, Brinn MP, Esterman AJ, Assendelft WJJ, et al. Training health professionals in smoking cessation. Cochrane Database of Systematic Reviews. 2012;(5). CD000214. http://dx.doi.org/10.1002/14651858.CD000214.pub2.

7. Aveyard P, West R. Managing smoke cessation. BMJ. 2007;335:37-41. http://dx.doi.org/10.1136/bmj.39252.591806.47.

8. Johns TL, Lawrence E, Martini LE, Dunn GE, Thompson ZJ, Zwygart K. Smoking cessation in Family Medicine: effects of an area health education center training program. J Grad Med Education. 2010;2(2):283-8. http://dx.doi.org/10.4300/JGME-D-10-00043.1.

9. Aveyard P, Begh R, Parsons A, West R. Brief opportunistic smoking cessation interventions: a systematic review and meta-analysis to compare advice to quit and offer of assistance. Addiction. 2011;107(6):1066-73. http://dx.doi.org/10.1111/j.1360-0443.2011.03770.x.

10. Reichert J, Araújo AJ, Gonçalves CMC, Godoy I, Chatkin JM, Sales MPU, et al. Diretrizes para cessação tabagismo - 2008. J Bras Pneumol. 2008;34(10):845-80. http://dx.doi.org/10.1590/S1806-37132008001000014.

11. Spangler JG, George G, Foley KL, Crandall SJ. Tobacco intervention training: current efforts and gaps in US medical schools. JAMA. 2002;288(9):1102-1109. http://dx.doi.org/10.1001/jama.288.9.1102.

12. Eddy, DM. David Eddy ranks the tests. Harv Healh Lett 1992;17(suppl.):10-11.

13. Murray RP, Connett JE, Rand CS, Pan W, Anthonisen NR. Persistence of the effect of the Lung Health Study (LHS) smoking intervention over eleven years. Prev Med. 2002;35(4):314-19. http://dx.doi.org/10.1006/pmed.2002.1087.

14. Stead LF, Lancaster T. Group behavior therapy programmes for smoking cessation. Cochrane Database of Systematic Reviews. 2005;(2). CD001007. http://dx.doi.org/10.1002/14651858.CD001007.pub2.

15. Leung L, Neufeld T, Marin S. Effect of self-administered auricular acupressure on smoking cessation - a pilot study. BMC Complement Altern Med. 2012;12(11):1-6. http://dx.doi.org/10.1186/1472-6882-12-11.

16. Ussher MH, Taylor A, Faulkner G. Exercise interventions for smoking cessation. Cochrane Database of Systematic Reviews. $2012 ;(1)$. CD002295. http://dx.doi.org/10.1002/14651858.CD002295.pub4.

17. Audrain-McGovern J, Benowitz, JL. Cigarette smoking, nicotine, and body weight. Clin Pharmacol Ther. 2011;90:(1):164-68. http://dx.doi.org/10.1038/clpt.2011.105.

18. Els C, Kunyk D, Sidhu H. Smoking cessation and neuropsychiatric adverse events: Are family physicians caught between a rock and a hard place? Can Fam Physician. 2011;57:647-49.

19. Ministério da Saúde (BR), Instituto Nacional do Câncer (INCA), Coordenação de Prevenção e Vigilância (CONPREV). Abordagem e tratamento do fumante - consenso 2001. Rio de Janeiro: INCA; 2001. 